\title{
Holographic grating formation in a colloidal suspension of silver nanoparticles
}

\author{
James R. Adleman \\ Department of Electrical Engineering, California Institute of Technology, Pasadena, California 91125 \\ Helge A. Eggert and Karsten Buse \\ Institute of Physics, University of Bonn, Wegelerstrasse 8, D-53115 Bonn, Germany \\ Demetri Psaltis \\ Department of Electrical Engineering, California Institute of Technology, Pasadena, California 91125
}

\begin{abstract}
Received October 7, 2005; revised November 9, 2005; accepted November 9, 2005; posted November 14, 2005 (Doc. ID 65235)
Holographic gratings are recorded in colloidal suspensions of silver nanoparticles by utilizing interfering nanosecond pulses. The diffraction efficiency is measured with continuous-wave light. An instantaneous response together with a transient grating are observed: the nanoparticles absorb the pump light and heat up. Heat is transferred to the solvent, and a delayed thermal grating appears. The final decay time constant of this grating depends quadratically on the period length and has a typical value of $1 \mu \mathrm{s}$ for grating spacings of several micrometers. (C) 2006 Optical Society of America

OCIS codes: $090.7330,160.4330,190.3970$.
\end{abstract}

Colloidal suspensions of metal nanoparticles have interesting optical properties. The high third-order nonlinear response of the nanoparticles for wavelengths close to the surface plasmon resonance has been the subject of intense studies. ${ }^{1-3}$ Since the absorption due to the nanoparticles also causes heating of the surrounding solvent, a temperature pattern can be created by means of structured illumination. This leads to a thermal change of the refractive index as well as to a particle redistribution caused by the so-called Soret effect. ${ }^{4}$ These thermal forces or the use of optical tweezers can allow on-demand configuration of, e.g., microfluidic devices or holographic Bragg filters. ${ }^{5}$ An advantage of colloidal suspensions compared with fabrication of structures in doped glasses is that the nanoparticles in suspension are easily reconfigurable. ${ }^{6}$ By choosing the right illumination conditions it is possible not only to transport and structure the nanoparticle distribution but also to get a patterned nonlinear optical response.

In this Letter we study the optical and thermal response as well as the transport properties of colloidal suspensions of silver nanoparticles. In these materials the light-matter interaction is still not completely understood, especially on the nanosecond time scale. ${ }^{7}$ We probe the material response by means of a simple holographic experiment. Elementary holographic gratings are recorded with nanosecond pulses in samples of different volume fractions of nanoparticles. The diffraction efficiency of these gratings is monitored with continuous-wave light. Maximum diffraction efficiencies of around 3\% were measured, corresponding to phase gratings with refractive index contrasts of approximately $1 \times 10^{-5}$.

Experiments are performed with chemically synthesized silver spheres with a mean diameter of $\approx 3.8 \mathrm{~nm}$ suspended in toluene. The surface plasmon resonance has its maximum around $435 \mathrm{~nm}$. Various concentrations between $3.5 \times 10^{14} \mathrm{~cm}^{-3}$ and 0.7 $\times 10^{14} \mathrm{~cm}^{-3}$ (optical density, OD, at $532 \mathrm{~nm}$ of $1.1-$ 0.22 ) were produced by dilution. The particle size is determined from the measured spectra by Mie theory calculations with a semiclassical correction, following Collier. ${ }^{8}$ To record a holographic grating, interference of two plane waves is used. Frequency-doubled pulses from a Nd:YAG laser (wavelength $\lambda=532 \mathrm{~nm}$, intensity $I=10 \mathrm{GW} / \mathrm{m}^{2}$, pulse duration $\tau=6 \mathrm{~ns}$, FWHM beam diameter $6 \mathrm{~mm}$ ) are split into two parts of equal intensity that overlap and interfere inside a $4 \mathrm{~mm}$ thick and $10 \mathrm{~mm}$ broad cuvette filled with the nanoparticle suspension. The angle between the pulses can be adjusted such that grating periods $\Lambda$ between 1 and $2.5 \mu \mathrm{m}$ are possible. The induced grating is observed by diffracting a Bragg-matched, continuous-wave beam of a laser diode at $\lambda=785 \mathrm{~nm}$ with a beam diameter of $2 \mathrm{~mm}$. All three beams are $s$ polarized. A fast photodiode with a rise time of $1 \mathrm{~ns}$ and a digital oscilloscope (bandwidth $1 \mathrm{GHz}$ ) are used to capture the time evolution of the diffracted signal. A bandpass filter ( $800 \mathrm{~nm}, 40 \mathrm{~nm}$ FWHM) at the read-out wavelength blocks the pump light at the detector. Between each recording experiment the sample is allowed to cool for at least $1 \mathrm{~min}$ to avoid boiling of the solvent. This time is also sufficient to flatten out a possible concentration distribution of the nanoparticles. ${ }^{4}$ The absorption spectra of all samples were measured before and after each experiment, and no change was observed. This indicates that the pulsed illumination does not lead to agglomeration or shape changes of the nanoparticles.

The diffraction efficiency of the grating depends strongly on the concentration of the nanoparticles and on the light intensity. At lower intensities the diffracted signal shows an abrupt rise time of around 


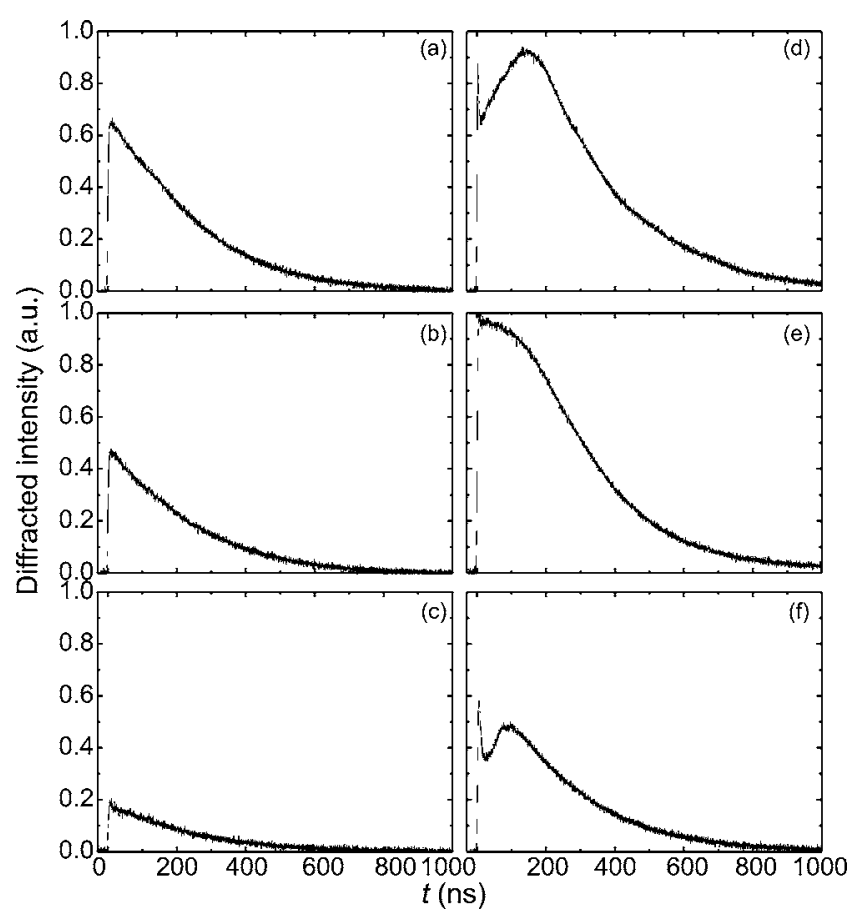

Fig. 1. Intensity of the diffracted beam versus time $t$ for different particle concentrations. Pump intensities (a)-(c) $I=4.6 \mathrm{GW} / \mathrm{m}^{2}$ and (d)-(f) $I=10 \mathrm{GW} / \mathrm{m}^{2}$. Particle concentrations (a), (d) $3.47 \times 10^{14} \mathrm{~cm}^{3}$ (OD 1.1), (b), (e) 1.74 $\times 10^{14} \mathrm{~cm}^{3}$ (OD 0.55), and (c), (f) $0.7 \times 10^{14} \mathrm{~cm}^{3}$ (OD 0.22).

$6 \mathrm{~ns}$, and then a monoexponential decay with a time constant of $\tau=220 \mathrm{~ns}$. This decay time constant does not vary with concentration [Figs. 1(a)-1(c)].

At the highest intensities measured, the time evolution of the diffracted signal is qualitatively different for different concentrations [Figs. 1(d)-1(f)]. At the two lowest concentrations (OD 0.32, 0.22) the initial rise still occurs in $6 \mathrm{~ns}$, followed by a dip and a slower second rise that peaks at around $150 \mathrm{~ns}$. This is followed by the decay of the grating, with the same time constant as before [Fig. 1(f)].

At the intermediate concentration (OD 0.55) the first minimum is not present. However there is still a slow rise and decay of the signal [Fig. 1(e)]. Finally, at the highest concentrations and intensities, an initial peak is again present in the diffracted efficiency curve. The magnitude of the first maximum is now smaller than the second. The rise and fall times of the first peak are faster, at around $3 \mathrm{~ns}$ [Fig. 1(d)].

Comparing measurements at different intensities in single samples, we see a smooth evolution in the behavior of the diffracted signal. At low intensities a monoexponential decay is seen; as the intensity increases, the faster transient effects grow in magnitude. The position of the second maximum shifts to later times with increasing intensity (Fig. 2).

Variation of the grating period length $\Lambda$ between 1 and $2.4 \mu \mathrm{m}$ has no influence on the first peak or on the buildup of the second one. However, the amplitude of the grating decays slower with increasing grating spacings, clearly pointing to thermal effects (Fig. 3). Thus the final time evolution of the diffraction efficiency can be explained by the presence of a thermal grating in the solvent: energy absorbed by the nanoparticles heats the solvent. Since each nanoparticle heats a spherical volume of solution with a diameter equal to the mean interparticle spacing (150-250 nm), there can be some delay in building up a smooth sinusoidal temperature distribution. In our experiments a strong thermal grating has been established by $200 \mathrm{~ns}$ after the recording pulses. The decay afterward is governed solely by thermal conductivity and can be used to determine the thermal diffusivity of the sample. ${ }^{9,10}$ The temperature amplitude $\Delta T$ of the thermal grating decays exponentially,

$$
\Delta T=T_{0} \exp \left(-t / \tau_{\text {th }}\right),
$$

with the time constant

$$
\tau_{\text {th }}=\Lambda^{2} / 4 \pi^{2} \kappa .
$$

Here $\kappa$ is the thermal diffusivity of the solvent. The refractive index change of the solvent is proportional to $\Delta T$, and hence, according to the coupled-wave theory, ${ }^{11}$ the diffraction efficiency is proportional to the square of the temperature change for small diffraction efficiencies.

From the fit in Fig. 3 and taking into account the factor of 2 between the time constants for the diffraction efficiency and the refractive index change for small diffraction efficiencies, we can then calculate the thermal diffusivity of the solvent as 7.27 $\times 10^{-8} \mathrm{~m}^{2} / \mathrm{s}$. This is in good agreement with the literature value of $8.85 \times 10^{-8} \mathrm{~m}^{2} / \mathrm{s} .{ }^{12}$

The temporal evolution for times less than $200 \mathrm{~ns}$ is more complex. At low concentrations and intensities the diffraction efficiencies are consistent with a simple thermal grating. However, as the intensity increases, another transient signal appears. Also, the maximum diffraction efficiency scales linearly with intensity, instead of quadratically. The disappearance of the dip between the first and the second maximum for intermediate concentrations, as well as the change in position and magnitude of the second

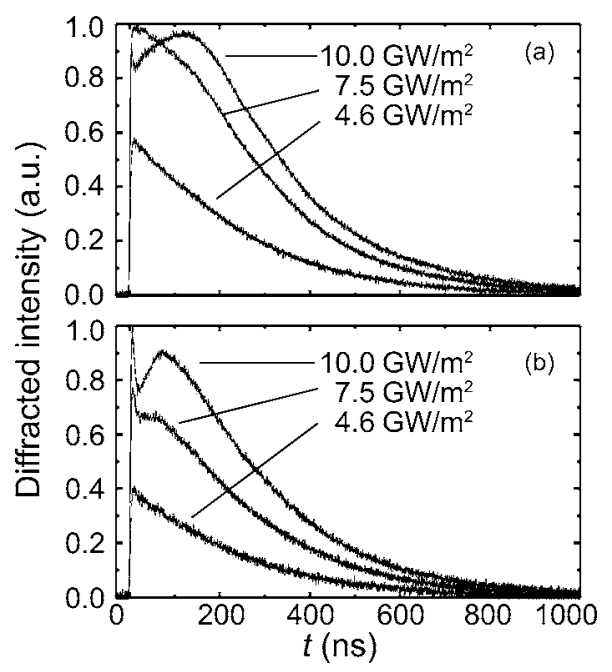

Fig. 2. Diffracted intensity versus time $t$ for three different pump intensities in samples with particle concentrations of (a) $2.4 \times 10^{14} \mathrm{~cm}^{3} \quad(\mathrm{OD}=0.76)$ and (b) 1.07 $\times 10^{14} \mathrm{~cm}^{3}(\mathrm{OD}=0.34)$. 


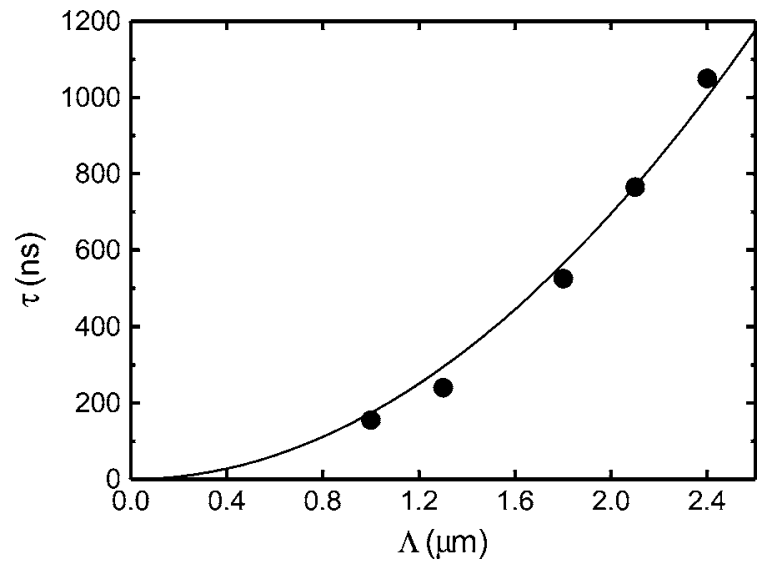

Fig. 3. Time constant $\tau$ of the decay of the diffraction efficiency versus grating period $\Lambda$ of the thermal grating. The solid curve is a quadratic fit according to the equation $\tau$ $=a \times \Lambda^{2}$.

maximum relative to the first, suggests that there is more than one physical effect operating on the short time scale (Fig. 1).

Different mechanisms for optical nonlinear effects in solutions of metal nanoparticles are known. ${ }^{13}$ Changes of the polarizability of the particles due to hot electrons and electron-dipole transitions are instantaneous in the nanosecond regime. Also, the field enhancement in the vicinity of the particles due to the surface plasmon resonance may lead to a high instantaneous Kerr nonlinearity in the solvent. Measurements with femtosecond and picosecond pulses show positive nonlinear refractive index changes of either the nanoparticles or the solvent. Heating the solvent leads to thermal and thus negative changes of the refractive index.

Despite the fast thermalization of absorbed energy by the metal nanoparticles, it has been shown that the heat transfer from nanoparticles excited by nanosecond pulses to the solvent can last for several tens of nanoseconds. This behavior is strongly influenced by both particle size and concentration. ${ }^{7}$ This diffusion of thermal energy into the solvent could be the mechanism responsible for the discrepancies in the temporal evolution of the first peak and valley. A thermally induced modification of the optical properties of the particles can perhaps lead to a change of the fast nonlinear response. But another explanation may be possible: the absorbed energy may be sufficient to boil a small shell of the solvent around each nanoparticle, thus leading to strong refractive index changes, resulting in a change of the surface plasmon resonance and in light scattering at the phase boundary in the solvent. ${ }^{4}$ In either case, the refractive index grating shortly after the pump pulse could be described as the sum of an instantaneous Kerr effect, a fast-forming simple thermal grating accounting for the monoexponential trend, and a longer-lived delayed grating due to the thermal changes in the particles and the solvent. Further investigation is necessary to determine the exact parameters of these gratings.

In conclusion, we have shown that different nonlinear effects compete when a colloidal suspension of silver nanoparticles is illuminated with $6 \mathrm{~ns}$ laser pulses. The shorter-time-scale behavior of these systems is the sum of thermal and electronic nonlinearities, which cause dramatic differences in properties of holographic gratings written at different particle concentrations and pump powers.

Financial support of the Deutsche Telekom AG, the Deutsche Forschungsgemeinschaft, and the DARPA center for optofluidic integration is gratefully acknowledged.

\section{References}

1. S. Link and M. A. El-Sayed, Annu. Rev. Phys. Chem. 54, 331 (2003).

2. C. Voisin, N. D. Fatti, D. Christofilos, and F. Vallée, J. Phys. Chem. B 105, 2264 (2001).

3. C. Flytzanis, J. Phys. B 38, 661 (2005).

4. J. Lenglet, A. Bourdon, J. C. Bacri, and G. Demouchy, Phys. Rev. E 65, 031408 (2002).

5. A. Terray, J. Oakey, and D. W. M. Marra, Appl. Phys. Lett. 81, 1555 (2002).

6. M. Rosenbluh, I. Antonov, D. Ianetz, Y. Kaganovskii, and A. A. Lipovskii, Opt. Mater. 24, 401 (2003).

7. M. Rashidi-Huyeh and B. Palpant, J. Appl. Phys. 96, 4475 (2004).

8. C. P. Collier, "Design and characterization of a reversible metal-insulator transition in silver quantum dot monolayers," Ph.D. dissertation (University of California, Berkeley, 1998).

9. H. Eichler, G. Salje, and H. Stahl, J. Appl. Phys. 44, 5383 (1973).

10. Y. Nagasaka, T. Hatakeyama, M. Okuda, and A. Nagashima, Rev. Sci. Instrum. 59, 1156 (1988).

11. H. Kogelnik, Bell Syst. Tech. J. 48, 2909 (1969).

12. D. R. Lide, ed., CRC Handbook of Chemistry and Physics (Taylor \& Francis, 2005).

13. H. B. Liao, R. F. Xiao, J. S. Fu, H. Wang, K. S. Wong, and G. K. L. Wong, Opt. Lett. 23, 388 (1998).

14. F. Lang, P. Leiderer, and S. Georgiou, Appl. Phys. Lett. 85, 2759 (2004). 